\title{
The Montreal Cognitive Assessment (MoCA) with a double threshold: improving the MoCA for triaging patients in need of a neuropsychological assessment.
}

\author{
Céraud M. F. C. Dautzenberg, ${ }^{1,2}$ (1) Jeroen G. Lijmer, ${ }^{3}$ and Aartjan T. F. Beekman² \\ ${ }^{1}$ Altrecht Institute for Mental Health Care, Old Age Psychiatry, Soestwetering 1, 3543AZ Utrecht, The Netherlands \\ ${ }^{2}$ Department of Psychiatry, Amsterdam UMC, location VUmc and GGZ inGeest, Oldenaller 1, $1081 \mathrm{HF}$, Amsterdam, The Netherlands \\ ${ }^{3}$ Department of Psychiatry, OLVG Hospital, Postbus 95500, 1090 HM, Amsterdam, The Netherlands
}

Objectives: Diagnosis of patients suspected of mild dementia (MD) is a challenge and patient numbers continue to rise. A short test triaging patients in need of a neuropsychological assessment (NPA) is welcome. The Montreal cognitive assessment (MoCA) has high sensitivity at the original cutoff $<26$ for $\mathrm{MD}$, but results in too many false-positive (FP) referrals in clinical practice (low specificity). A cutoff that finds all patients at high risk of MD without referring to many patients not (yet) in need of an NPA is needed. A difficulty is who is to be considered at risk, as definitions for disease (e.g. MD) do not always define health at the same time and thereby create subthreshold disorders.

Design: In this study, we compared different selection strategies to efficiently identify patients in need of an NPA. Using the MoCA with a double threshold tackles the dilemma of increasing the specificity without decreasing the sensitivity and creates the opportunity to distinguish the clinical (MD) and subclinical (MCI) state and hence to get their appropriate policy.

Setting/participants: Patients referred to old-age psychiatry suspected of cognitive impairment that could benefit from an NPA $(n=693)$.

Results: The optimal strategy was a two-stage selection process using the MoCA with a double threshold as an add-on after initial assessment. By selecting who is likely to have dementia and should be assessed further $(\mathrm{MoCA}<21)$, who should be discharged $(\geq 26)$, and who's course should be monitored actively as they are at increased risk $(21<26)$.

Conclusion: By using two cutoffs, the clinical value of the MoCA improved for triaging. A double-threshold MoCA not only gave the best results; accuracy, PPV, NPV, and reducing FP referrals by $65 \%$, still correctly triaging most $\mathrm{MD}$ patients. It also identified most MCIs whose intermediate state justifies active monitoring.

Key words: MoCA, double threshold, triaging, cognitive impairment, old-age psychiatry, memory clinic, subthreshold disorders, intermediate state

\section{Introduction}

More diagnostic effort is recommended by the Alzheimer's society because early recognition of dementia allows for timely interventions and better quality of life for the patients (Borson et al., 2013). However, the (clinical) reality has its limitations.

The diagnosis of patients with suspected mild dementia (MD) is challenging, and the number of patients continues to rise.

Correspondence should be addressed to: Géraud M. F. C. Dautzenberg, Altrecht Institute for Mental Health, Department of Old Age Psychaitry, Soestwetering 1, 3543AZ, Utrecht, The Netherlands. Phone: + 3130 2297600; Fax: + 3130 3103342. Email: g.dautzenberg@altrecht.nl. Received 20 Jul 2020; revision requested 23 Oct 2020; revised version received 30 Mar 2021; accepted 18 Apr 2021. First published online 31 August 2021.
It is difficult to differentiate who has MD based on anamnesis alone. Subjective complaints and reports from informants often do not correspond to objective impairments (Schouws et al., 2012; Pendlebury et al., 2015; Ryu et al., 2020).

Specialized diagnostic facilities are needed but will become overloaded by the number of referred patients in the near future. Most countries already have diagnostic challenges (Alzheimer's Disease International, 2018), including a lack of financial or staff resources for a time-consuming comprehensive neuropsychological assessment (NPA). An accurate short screening test to identify patients with a (high) risk of MD, i.e. those in need of an $\mathrm{NPA}$, is therefore necessary. A difficulty is who is to be considered at risk as definitions for disease (e.g. 
MD) do not always define health at the same time and thereby create subthreshold disorders (Helmchen and Linden, 2000). Cognitive functioning is a state on a continuum with dementia on one end and no cognitive impairment (NoCI) on the other end of the extremes. Classifications define these states, therefore creating double thresholds. In-between, there is an area in which the patient is in an intermediate state and at risk, e.g. mild cognitive impairment (MCI), of which approximately $40 \%$ worsens, $40 \%$ stabilizes, and $20 \%$ recovers (Gauthier et al., 2006; Julayanont et al., 2014; Canevelli et al., 2016).

Given the wide range of outcomes of MCI and the large numbers involved, it is essential to be able to differentiate patients with MCI from those with MD and NoCI (Gauthier et al., 2006). In particular, in an old-age psychiatry setting, there is a high correlation between psychiatric conditions (American Psychiatric Association, 2013) (including psychotropic medication and substance abuse) and MCI that does not necessarily worsen over time (Julayanont et al., 2014). These MCI cases deserve their own policy. An elaborate diagnostic route (including biomarkers/MRI) is often not yet necessary, but they should not be discharged either. An NPA comes to mind as a compromise. However, limited resources warrant the restraint of falsepositive (FP) referrals for an NPA to avoid potential harm due to unnecessary emotional and financial burden (Borson et al., 2013; Burn et al., 2018; Davis et al., 2015). Although early identification of neurocognitive disorders is advocated spending resources wisely is as important, giving the ones the most in need priority (Alzheimer's Disease International 2018; Borson et al., 2013). This implies that patients at highest risk (taking into account the age or speed of onset in combination with the degree of impairment) should be referred for an elaborate specialized diagnostic route. The cognitive functioning of patients considered to be at lower risk (e.g. with psychiatric disorders) should be assessed in the best available way, depending on the resources. When the scarce and time-consuming gold standard, i.e. an NPA, is less available the assessment of the cognitive functions could be done with a short, validated test. In our opinion, this should include reassessment with this test, as it is easy to perform and takes limited time to administer, i.e. active monitoring. Therefore, it is important to use screening instruments that can detect both $\mathrm{MD}$ and MCI.

The Montreal Cognitive Assessment (MoCA) was developed as a short screening tool for MCI and MD (Nasreddine et al., 2005) and validated in at least 35 different languages and even more settings. Most of these studies can be found on the MoCA-test website (mocatest.org). At the original proposed cutoff of $<26$, the sensitivity for correctly screening patients with MCI $(90 \%)$ and MD (100\%) is very good (Nasreddine et al., 2005). Although it has been repeatedly shown to be superior to the MMSE in identifying MCI (Folstein, et al., 1975; Pinto et al., 2019), the MoCA still has its limitations as a triaging tool. Its ability to identify people with NoCI (specificity) is criticised in clinical practice because specificity varies due to clinical and demographic reasons (Davis et al., 2015). Frequently reported examples are age, education, rural environment, ethnic or cultural background (including race in some countries), substance abuse, and psychiatric diseases (mocatest.org). It is repeatedly suggested to lower the cutoff with higher specificity as a result (O'Driscoll and Shaikh, 2017; Carson et al. 2018; Dautzenberg et al., 2020). Nevertheless, the MoCA with a lower cutoff is still not suitable for identifying $\mathrm{MD}$ as a stand-alone assessment of referred patients to an old-age psychiatric clinic (Dautzenberg et al. 2020; Korsnes, 2020), or as an assessment of referred patients to its memory clinic (Smith et al. 2007; Dautzenberg et al., 2021) because the positive predictive value (PPV) is never sufficient (Carson, et al., 2018). Its high sensitivity makes it a good screener, finding most $\mathrm{MD}$ patients. The high negative predictive value (NPV) for appropriately discharging NoCI patients, is promising, although for triaging those who need a scarce NPA, moderate specificity gives too many FPs.

Double cutoffs are reported in the literature as a solution by using one threshold for health and one for disease (Batelaan et al., 2007; Swartz et al., 2016; Landsheer, 2020; Thomann et al., 2020). Especially where classifications create subthreshold disorders, regardless of whether these are disorders in their own right or are merely (minor) forms of major disorders (Batelaan et al., 2007). Either way MCI is not (yet) dementia.

A double-threshold MoCA offers the possibility to distinguish clinical and subclinical states according to their appropriate domain and thus to implement different policies. Previous studies have shown that almost no patients with MD or even MCI will score $\geq 26$, the originally proposed cutoff of the MoCA, therefore indicating health (i.e. NoCI) (Nasreddine et al., 2005; Davis et al., 2015; Carson et al., 2018; Dautzenberg et al., 2020, 2021; Korsnes, 2020). The number of FPs below this cutoff is too large to have a full work-up. 'Reducing the risk of FP is important (Davis et al., 2015). In a memory clinic setting, half of the depressed patients scored below 26 (Blair et al., 2016; Dautzenberg et al., 2021). Other studies showed that the majority 
of patients with affective, psychotic, or neurotic disorders scored between 20 and 26 on the MoCA, while the majority of the organic disorders scored <19 (Gierus and Mosiolek, 2015; Dautzenberg et al., 2021; Korsnes, 2020). By using a lower (second) cutoff for referral (using the highest Youden index for MD in this cohort; <21) (Dautzenberg et al., 2020), FPs will decrease, but this will also increase the FNs. This could be compensated for by actively monitoring all these patients with a score from 21 to 26 , reducing unnecessary referrals (FP), but still allowing those patients at high risk of MD to be monitored (FN).

A recent study by Landsheer demonstrated that using a double threshold for the MoCA improves clinical classification and that using an uncertainty interval (21 to 26) reduces the effect of prevalence on MoCA performance (Landsheer, 2020).

Other studies on double thresholds aimed to improve classification accuracy by stratifying the population based on normative data (Oren et al., 2014; Tan et al., 2014; Borland et al., 2017) or stratifying the outcome, of certain or uncertain test results (Swartz et al., 2016; Landsheer, 2020; Thomann et al., 2020). They do not separate the three distinct cognitive states, i.e. $M D$ versus MCI versus NoCI.

In our study, however, we wanted to introduce three policies, matching the three diagnostic entities of cognitive functioning, to improve the MoCA's potential as a triaging tool.

Although the use of the MoCA in this way feels intuitive, to our knowledge, no results have been presented before on the MoCA with a double threshold separating all three distinct stages and analyzing the consequences of subsequent policies.

Especially in old-age psychiatry, there are many inconclusive MoCA scores due to age $(60+)$ and psychiatric comorbidities (including psychotropic medication), i.e. from 21 to 26 . Therefore, we studied the policy of 'active monitoring' in this population as an intermediate option.

We used data from our cohort of an old-age psychiatric setting (Dautzenberg et al., 2020, 2021), where referrals, at least in the Netherlands, include patients with cognitive, behavioral, and psychiatric symptoms, that may result from neurodegenerative diseases, but also from other psychiatric disorders. The standard is that after an initial assessment, it is decided who could benefit from an extensive cognitive diagnostic route at our memory clinic.

We aim to demonstrate the advantages of using a double-threshold MoCA to triage patients in need of an NPA. Therefore, we compare different selection strategies, including the double threshold, to efficiently select patients in need of an NPA (i.e. MD), those who are not (NoCI), and patients who should be actively monitored (MCI). We rate the strategies according to their accuracy and the number of referrals for an NPA that result in as few FNs as possible.

The compared selection strategies for referral to an NPA are as follows: an initial assessment only (without the use of an objective test, i.e. the MoCA), the MoCA as a stand-alone (i.e. without clinical judgment), or the MoCA as an add-on after the initial assessment (i.e. as a two-stage screener). The MoCA strategies are compared when using single and double thresholds.

\section{Methods}

\section{Patients}

The cohort $(n=693)$ was taken from a previously reported validation study of the MoCA for patients referred to an old-age psychiatric service in Utrecht, the Netherlands, as described in detail elsewhere (Dautzenberg et al., 2020). In short, all newly referred patients to our clinic were eligible for the study if they were capable of giving written informed consent. Therefore, patients with severe dementia (Global Deterioration Scale (GDS) $\geq 6$ ) (Reisberg et al., 1982) and Behavioral and Psychological Symptoms of Dementia (BPSD) as a reason for referral and compulsory referrals were not eligible $(n=1337)$. To resemble a clinical screening population, Standards for Reporting Diagnostic Accuracy (STARD/ STARDdem) (Noel-Storr et al., 2014; Bossuyt et al., 2015) require we excluded patients with an obvious diagnosis of dementia (GDS $\geq 5$ ), a recent history of substance abuse ( $<1$ year), delirium $(<6$ months), or acquired brain injury including CVA or TIA $(n=174)$. The patients suspected of having cognitive impairment after the initial assessment were referred to our memory clinic $(n=290)$ (Figure 1). All of these patients underwent a comprehensive cognitive diagnostic route for cognitive impairment using a consensus-based diagnosis, following international criteria as a reference standard with an NPA and when applicable, CT/MRI- imaging and cerebrospinal fluid (CSF) analysis (CBO Geriatrie, 2014). They were classified as $\mathrm{MD}$, MCI (including psychiatric etiologies), or NoCI (including subjective complaints, mostly psychiatric patients without objective cognitive impairment).

For the strategic selection route comparison, we included all eligible referred patients $(n=693)$, including those not suspected of cognitive impairment $(n=403)$. The latter were followed for at least 2 years to compensate for not having 
Stand-alone

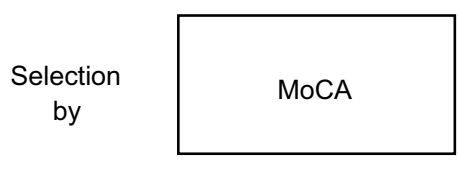

Referral

NPA
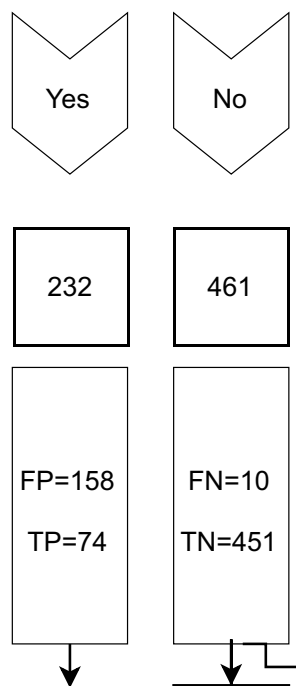

MD

False

pos/neg

True

pos/neg

$\mathrm{MCl}$

$\mathrm{No}-\mathrm{Cl}$

Action

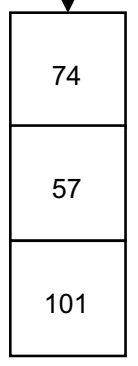

MoCA

$<21$

Reffered
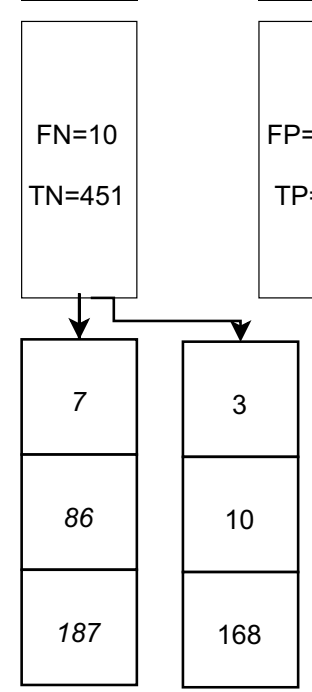

MoCA

$21<26$

+Monitoring

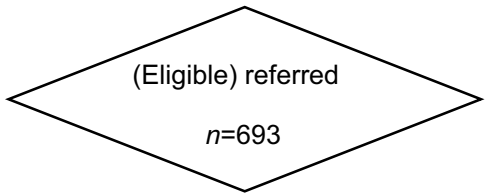

Add-on
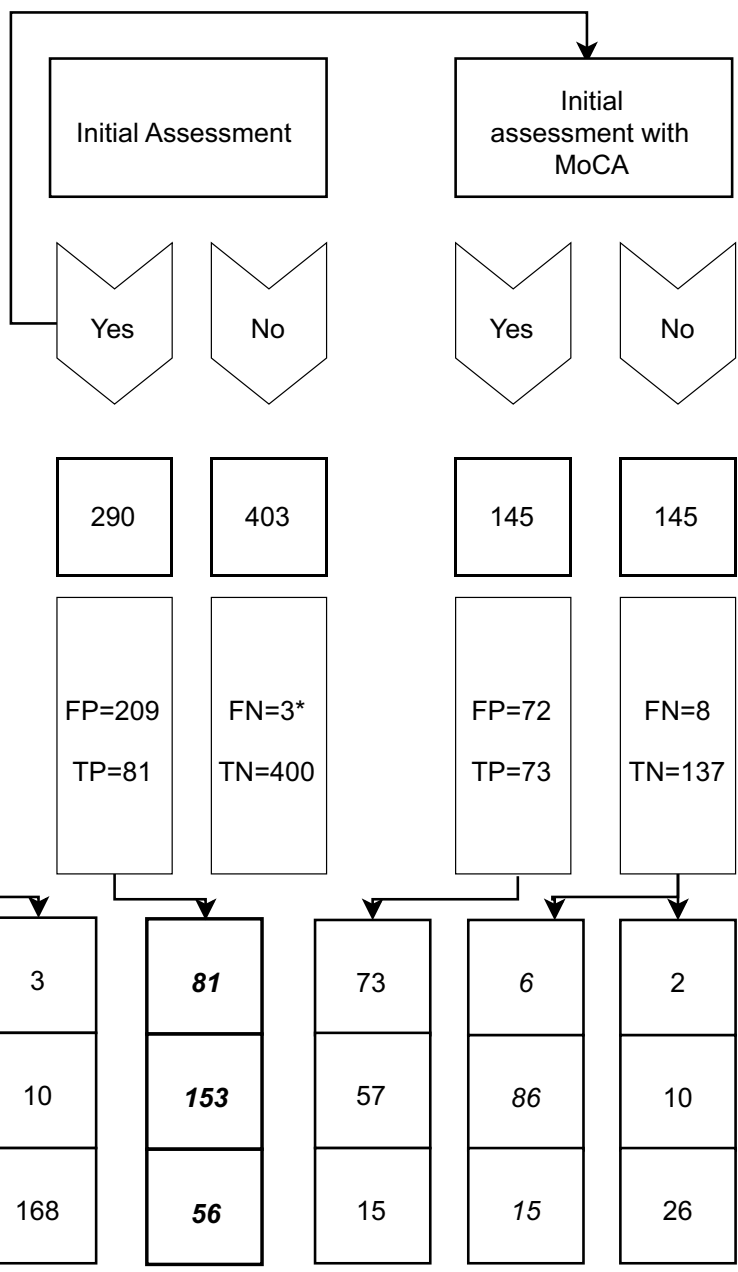

NPA

MoCA

MoCA

MoCA

$<21$

$21<26$

$\geq 26$

Referred +Monitoring None

\section{A $\quad$ B $\quad$ C}

D

E

F

G

Figure 1. Flowchart and results of the different selection strategies.

MoCA: Montreal Cognitive assessment.

NPA: comprehensive NeuroPsychological Assessment.

FP: False Positive; FN: False Negative; TP: True Positive; TN: True Negative.

*FN during follow up.

an NPA to exclude conversion to any DSM IV/5 (American Psychiatric Association, 2000, 2013) cognitive diagnoses after the initial assessment. Three of them were diagnosed with cognitive impairment during follow-up and were considered inconclusive as it is not certain if these impairments manifested before or after initial assessment. To be conservative, we classified these three as FN MD. 
All participants gave their informed consent. Data are available upon request.

\section{Measurements}

\section{Initial assessment}

The initial assessment was completed by old-age psychiatrists $(n=4$, having at least 8 years of practical experience in 2008) and included a laboratory test, medical, and functional history from a next of kin and an investigation of Instrumental Activities of Daily Living (IADL) performed by a psychiatric nurse practitioner with a home visit. The 15 -item Geriatric Depression Scale (GDS15) (Yesavage and Sheikh, 1986) and the Global Assessment of Functioning (GAF) (American Psychiatric Association, 2000) were also administered during this time.

\section{Diagnostic test}

All of the referred participants were assessed with an MoCA as soon as possible but within 3 months of referral by a trained psychiatric nurse practitioner. This was independent of the diagnostic procedure.

The MoCA consists of one page that covers the cognitive domains of executive function and visuospatial abilities, naming, short-term memory, attention and working memory, language, concentration, verbal abstraction, and orientation. It can be administered within 10 minutes and the maximum score is 30 , which indicates that no errors were made. Correction for low education effects was done, according to the instructions, by adding one point to the total of patients with 12 years of education or less. The suggested best cutoff for the diagnosis of dementia was a score of $21 \quad(<21)$ and $<26$ for MCI, in both old-age psychiatry and memory clinic settings (Dautzenberg et al., 2020, 2021).

\section{Reference test}

The reference test was the diagnosis determined at multidisciplinary meetings, including an old-age psychiatrist, neuropsychologist, and geriatrician. The diagnosis of MD, MCI, or NoCI was supported with at least a 4-h NPA. The NPA included multiple tests in the domains of memory, attention, executive function, fluid intelligence, and language capacities (for details please see Dautzenberg et al., 2020). The diagnoses were made in consensus and in accordance with the MCI criteria as proposed by an international consortium (Winblad et al., 2004; Gauthier et al. 2006), or the Dutch guideline on dementia (CBO Geriatrie, 2014). This guideline covers the criteria of -DSM IV/5 and the international criteria for dementia. The results of the MoCA were not used to diagnose MCI or dementia.
The Dutch translation of the DSM 5 was introduced in the Netherlands at the end of the study of the cohort (2017). All patients were classified according to DSM IV for the purpose of this study.

\section{Statistical analyses}

\section{Statistics}

The demographic results (Table 1 ) were compared within the patients with MD, MCI, or NoCI using Statistical Package for the Social Sciences (SPSS, version 22; SPSS Inc., Chicago, IL); Chi2 test to compare sex and education. ANOVA was used to compare age, GAF, GDS15, and MoCA scores followed by a least significant difference post hoc test.

The previously reported area under the curve calculations using receiver operating characteristic analysis were used to find the best cutoff scores (Dautzenberg et al., 2020, 2021) for both settings (Table 2).

We reported the FPs, false negatives (FNs), true positives (TPs), and true negatives (TNs) of the different selection strategies to judge the clinical effects (Figure 1). The positive predictive value $(\mathrm{PPV}=\mathrm{TP} /$ $(\mathrm{TP}+\mathrm{FP}))$, negative predictive value $(\mathrm{NPV}=\mathrm{TN} /$ $(\mathrm{TN}+\mathrm{FN}))$, and accuracy $(\mathrm{ACC}=(\mathrm{TP}+\mathrm{TN}) /(\mathrm{TP}+$ $\mathrm{TN}+\mathrm{FP}+\mathrm{FN}$ )) were calculated (Table 3$)$. However, with these indicators, it is impossible to weigh the FN and FP rates separately, which is a disadvantage, and absolute quantities can provide more insight when diagnostic routes are compared (Glas et al., 2003). Therefore, we also expressed the results of the selection strategies in absolute numbers (Figure 1, Tables 3 and 4) of patients who were to be referred (Figure 1; columns A, E), those who were to be observed (B, F), who were not $(C, G)$, and the reference diagnoses $(D)$. As the purpose of the study is to reduce the number of FP referrals without discharge a MD patient, we considered the observed MD, MCI, and NoCI as TP, TN, and TN, respectively. The calculation of the indicators for the two-stage strategy included the effects of the initial assessment (e.g. adding the 400TN and 3*FN to columns E, F, G).

\section{Results}

\section{Demographic findings}

The main demographic and clinical characteristics of the study group are listed in Table 1.

The significant differences in age and sex are representative of the demographics of an old-age psychiatry setting and are substantiated elsewhere (Dautzenberg et al., 2020).

The GAF score was the highest in the MCI group, as expected because these patients have the 
Table 1. key demographic and clinical characteristics

\begin{tabular}{|c|c|c|c|c|c|}
\hline & DEMENTIA（A） & MCI (в) & NoCI (c) & TOTAL REFERRED & STATISTIC DIFFERENCE $\mathrm{P}<0.001$ \\
\hline Variable $/ n$ & 84 & 153 & 456 & 693 & \\
\hline Age & 77.3 & 73.9 & 71.3 & 72.5 & \\
\hline$(\mathrm{SD})$ & $(7,5)$ & $(8.0)$ & $(7.3)$ & $(7.8)$ & $a>b>c$ \\
\hline range & $59-94$ & $53-93$ & $58-92$ & $53-94$ & \\
\hline Education $<12(\%)$ & 52 & 53 & 43 & 47 & No sig. \\
\hline Sex F (\%) & 63 & 57 & 63 & 62 & No sig. \\
\hline GAF & 52 & 57 & 52 & 53.3 & $\mathrm{a}, \mathrm{c}<\mathrm{b}$ \\
\hline$(\mathrm{SD})$ & $(10.2)$ & $(12.8)$ & $(12.4)$ & (12.3) & \\
\hline range & $30-80$ & $20-90$ & 20-95 & 20-95 & \\
\hline GDS15 & 6.6 & 7.7 & 8.6 & 8.4 & No sig. \\
\hline$(\mathrm{SD})$ & $(4.9)$ & $(4.7)$ & $(4,3)$ & $(4.3)$ & \\
\hline range & $0-15$ & $0-14$ & $0-15$ & $0-15$ & \\
\hline MoCA & 16.5 & 20.9 & 23.5 & 22.1 & \\
\hline$(\mathrm{SD})$ & $(4.0)$ & $(3.8)$ & $(4.2)$ & $(4.7)$ & $\mathrm{a}<\mathrm{b}<\mathrm{c}$ \\
\hline range & $5-26$ & $3-28$ & $3-30$ & $3-30$ & \\
\hline
\end{tabular}

Groups a,b and c were compared with ANOVA, education and sex were compared with a Chi2 test.

MCI: Mild Cognitive Impairment; NoCI: No Cognitive Impairment; GAF: Global Assessment of Functioning; GDS15: Geriatric Depression Scale 15 question version; MoCA: Montreal Cognitive Assessment.

Table 2. Area Under the Curve between variations of groups and their sensitivity and specificity at cutoff scores 26 and 21, often used in literature. Stand-alone $(n=693)$ or add-on $(n=290)$

\begin{tabular}{|c|c|c|c|c|c|c|c|c|}
\hline \multirow[b]{2}{*}{ GROUPS } & & & \multirow[b]{2}{*}{ AUC } & \multirow[b]{2}{*}{$\mathrm{SD}$} & \multicolumn{2}{|c|}{ CUTOFF $<26$} & \multicolumn{2}{|c|}{ CUTOFF $<21$} \\
\hline & & & & & SENS & SPEC & SENS & SPEC \\
\hline $\mathrm{MD}$ & vs & NoDem $(n=693)$ & .865 & .018 & .975 & .292 & .901 & .740 \\
\hline $\mathrm{MD}$ & vs & NoDem $(n=290)$ & .830 & .026 & .975 & .173 & .901 & .654 \\
\hline MD & vs & MCI $(n=693)$ & .810 & .029 & .975 & .065 & .901 & .627 \\
\hline $\mathrm{MD}$ & vs & MCI $(n=290)$ & .810 & .029 & .975 & .065 & .901 & .627 \\
\hline $\mathrm{CI}$ & vs & NoCI $(n=693)$ & .765 & .018 & .949 & .368 & .556 & .778 \\
\hline CI & vs & NoCI $(n=290)$ & .770 & .040 & .949 & .473 & .556 & .727 \\
\hline MCI & vs & NoCI $(n=693)$ & .702 & .022 & .935 & .368 & .373 & .778 \\
\hline MCI & vs & NoCI $(n=290)$ & .707 & .048 & .935 & .473 & .373 & .727 \\
\hline
\end{tabular}

MD: Mild Dementia; NoDem: No Dementia (MCI + NoCI); MCI: Mild Cognitive impairment; NoCI: Referred patients no Cognitive Impairment; CI; Cognitive Impairment (Dem + MCI).

Stand-alone $(n=693)$ : all referred patients without judgement of initial assessment.

Add-on $(n=290)$ : only those patients suspected of CI after initial assessment. All referred to memory clinic.

Table 3. results of the selection strategies

\begin{tabular}{|c|c|c|c|c|c|c|c|c|c|c|c|}
\hline & & NPA & $\mathrm{FN}$ & $\mathrm{FP}$ & $\mathrm{TP}$ & $\mathrm{TN}$ & PPV & NPV & $\mathrm{ACC}$ & DIF & COLUMN FIGURE 1. \\
\hline & Cutoffs & $N$ & $N$ & $N$ & $N$ & $N$ & $\%$ & $\%$ & $\%$ & $\%$ & \\
\hline I.A. & $\mathbf{n} / \mathbf{a}$ & 290 & 3 & 209 & 81 & 400 & 27.9 & 99.3 & 69.4 & $\mathrm{n} / \mathrm{a}$ & $\mathrm{D}$ \\
\hline MoCA & $<26$ & 512 & 3 & 431 & 81 & 178 & 15.8 & 98.3 & 37.3 & +77 & $\mathrm{C}$ \\
\hline \multirow[t]{2}{*}{ S.A. } & $<21$ & 232 & $3+7$ & 158 & 74 & 451 & 31.9 & 97.8 & 75.8 & -20 & A \\
\hline & $21<26$ & 232 & 3 & 158 & $74+7^{\wedge}$ & 451 & 33.9 & 99.3 & 76.8 & -20 & B \\
\hline MoCA & $<26$ & 252 & $2+3^{*}$ & 173 & 79 & $36+400^{*}$ & 31.3 & 98.9 & 74.3 & -13 & $\mathrm{G}$ \\
\hline \multirow[t]{2}{*}{ A.O. } & $<21$ & 145 & $8+3^{*}$ & 72 & 73 & $137+400^{*}$ & 50.3 & 97.9 & 88.0 & -50 & $\mathrm{E}$ \\
\hline & $21<26$ & 145 & $2+3^{*}$ & 72 & $73+6^{\wedge}$ & $137+400^{*}$ & 52.3 & 99.1 & 88.9 & -50 & $\mathrm{~F}$ \\
\hline
\end{tabular}

*including FN/TN of Initial Assessment.

including the observation group.

I.A.: Initial Assessment. MoCA: Montreal Cognitive Assessment. S.A.: stand-alone. A.O.: add-on; NPA: referred for a Neuropsychological assessment. FN: False negative. FP: False Positive. TP; True Positive.TN: True Negative. PPV: Positive Predictive Value. NPV: Negative

Predictive Value. ACC: Accuracy. DIF: difference in referrals compared to I.A. 
Table 4. ( $a, b, c$ and $d)$ : cross tables of the different strategies

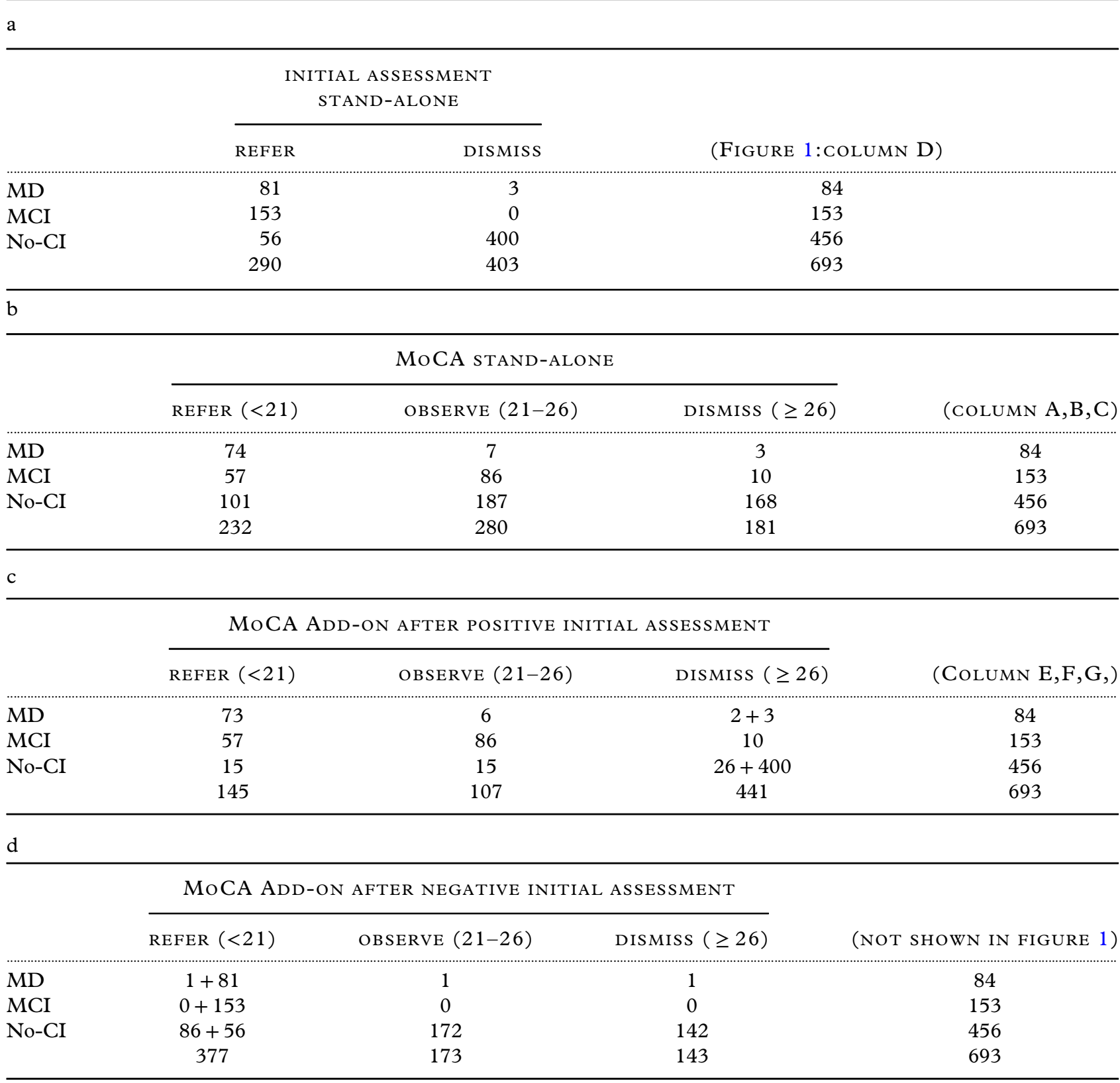

least severe symptoms, given the high number of MCI patients without any other psychiatric conditions $(50 \%)$, whereas almost all patients in the NoCI had one or more psychiatric diagnoses (Dautzenberg et al., 2020).

\section{Single threshold}

The initial assessment resulted in 290 referrals for NPA (Figure 1, column D). An accuracy of $69 \%$ was achieved for detecting MD (Table 3), with three FNs. Using a single MoCA threshold of $<21$ to select those requiring an NPA resulted in a decrease in referrals, but an increase in FNs compared to the initial assessment (columns A and E). However, this resulted in an improvement in their accuracy (Table 3). When the MoCA was used as an addon to screen the 'patients not suspected after initial assessment' ( $n=403$ : Table $4 \mathrm{~d}$, calculations not shown), the accuracy deteriorated to $57 \%$ by adding 87 referrals to the initial set of 290 referrals, resulting in a total of 377 referrals (including $297 \mathrm{FPs}$ ).

Comparing the PPVs and NPVs of the different selection strategies at a cutoff $<21$ for $M D$, we found a substantial increase in PPV and only a slight decrease in NPV when the MoCA was used as an add-on. 
When the cutoff was raised to $<26$ for detecting MD per strategy (columns C, G), the FNs decreased but with a substantial increase in referrals, which decreased the PPV and ACC.

\section{Double threshold}

The single threshold dichotomy reported above had a binary outcome: referral for NPA or no referral. This is not consistent with clinical practice, where an intermediate strategy of 'keeping the patient under observation' or 'active monitoring' is often used.

The use of two cutoff scores: $<21$ invitation for NPA, $21 \leq$ active monitoring $<26$; no follow-up $\geq 26$ gave a more differentiated result (columns $\mathrm{B}, \mathrm{F})$.

The double-threshold MoCA as an add-on for suspected patients after initial assessment (column F) resulted in $5\left(2+3^{*}\right)$ instead of $11 \mathrm{MD}$ (column E) patients with MD not undergoing a comprehensive diagnostic route, without the increase in FP referrals. This resulted in the highest accuracy (89\%), PPV (52\%), and NPV (99\%). However, it would mean that 107 patients need to be reassessed.

\section{Discussion}

Limited diagnostic resources and rising patient numbers present challenges in MD diagnostic procedures. It is necessary to differentiate patients to focus scarce diagnostic resources on those who need them most. Therefore, we compared different strategies, including a double-threshold MoCA. Our results confirm that an objective test can have added value. However, how and when the MoCA was used gave different results.

All the strategies we tested were able to find most of the patients with MD. Judgment by only the TP/ FN is not sufficient, as the FPs differs substantially (Figure 1/Table 3). Using merely initial assessment (column D) gave the highest TP of the compared strategies, but the high amount of FPs complicates the diagnostic route: still $42 \%$ of all assessed patients were referred for a comprehensive diagnostic route. This is probably because clinicians try to avoid FNs. Especially without the assistance of an objective test, clinicians tend to refer subthreshold states earlier.

The single cutoff MoCA strategy did not solve the (sub)threshold dilemma. Although the low PPV and the very high NPV underscore that only an MoCA score above the cutoff (i.e. a negative MoCA) should be considered reliable and thus suitable to adjust clinical judgment, i.e. initial assessment (Table 4d).

Using an MoCA as a second-stage screener with a score of 21 or higher (cutoff $<21$ ) to adjust initial assessment (column E) reduced the FP referrals for an NPA by $65 \%$ (to $n=72$ of 290) but increased the $\mathrm{FN}$ by 3.6 times to $13 \%$ of $\mathrm{MD}(n=11)$.

Using a double threshold with scores $<21$ identifying patients suitable for an NPA and $\geq 26$ for discharging patients, i.e. indicating patients do not need an NPA or reassessment, not only gave the best results but also achieved two goals simultaneously. Not only compensating for the increase in FNs by monitoring most of the missed MDs but also at-risk intermediate state patients (MCIs), without increasing the number of referrals.

How to classify the different strategic selection outcomes for the accuracy calculations can be debated and depends on the setting and its target disease. Introducing a double threshold together with an intermediate state raises the dilemma of what is to be considered TP/FP or TN/FN. As these cells only exist in a $2 \times 2$ classification table, in our study, we created a $3 \times 3$ table (Table $4 b, c, d$ ).

In addition to this theoretical classification problem, there is a clinical classification dilemma. There are multiple reasons to advocate (TP) or to be cautious (FP) with early MCI referrals, and the debate is ongoing. Because of limited access to NPAs in most countries or rural areas and because MCI can also consist of etiologies from which a patient can recover, we considered MCI an FP when a patient was referred for a comprehensive diagnostic route (therefore, MCI automatically became a $\mathrm{TN}$ for observation and discharging). Even though we understand that, with unlimited resources, one could consider (some of) the MCI-patients as TP when referred, as quality of life can improve by cognitive testing (Janssen et al., 2019). Identifying this intermediate state to actively monitor MCI without giving them this demanding diagnostic route is another justification for using a double threshold. Intuitively, we would consider $21 \leq \mathrm{MCI}<26$ as TP (for monitoring); however, technically this is not possible, as MCI is already labeled as an FP when a patient has a score $<21$.

For dementia, a short assessment that differentiates MD from MCI with certainty would be preferred, but such a test is still not available. This is also true for the MoCA, as our results showed that our best PPV is still too low (52\%) for a conclusive classification. Selecting those patients in need without missing one $\mathrm{MD}$ in the best way possible, without referring too many who are not (yet) in need of a memory clinic, is essential, i.e. triaging. These requirements were translated into our evaluation criteria of low absolute referral rates while still maintaining the highest possible sensitivity, i.e. no FNs and low FPs. Therefore, we judged the strategies by these values. 
Of the strategies selecting $\mathrm{MD}$, the double threshold add-on not only gave the highest accuracy (89\%), PPV (53\%), NPV (99\%), and the lowest FPs with still acceptable FNs, it also creates the opportunity to monitor MCI and seems the preferred selection route (Table 3, column F).

In addition to the accuracy calculations of our clinical example addressing the three cognitive entities as well as possible to their appropriate policy, as debated above, there are more arguments to be found in literature to use a double threshold for the MoCA.

First, a substantial number of MCI patients scoring below $<21$ on the MoCA are at very high risk of converting to $\mathrm{MD}$, while those above this cutoff are considerably less at risk (Smith et al., 2007; Julayanont et al., 2014; Dautzenberg et al., 2021).

Second, a double threshold for the MoCA also reduces the 'uncertain test scores' due to 'random classification errors'. These outcomes result from the distribution of the different diagnostic groups in the middle range MoCA scores (Landsheer, 2020; Thomann et al., 2020). By applying an uncertainty interval, as these MoCA scores are the most error prone, the PPV and NPV improve in the studied prevalence, and become less dependent on the setting. Even if their study objective was not to identify the subthreshold state, the implementation is similar; applying an (uncertainty) interval improves the accuracy of the MoCA.

Third, as mentioned in the introduction, several variables are found to be of importance in different clinical populations, and these can lead to an inflated rate of FPs particularly older age and lower education (Carson et al., 2018; Thomann et al., 2020). Lifestyle and physical activity are found to significantly influence MoCA scores even more than age and education (Ihara et al., 2013; Innocenti et al., 2017). Although education (Wong et al., 2015; Borland et al., 2017; Pinto et al., 2018), ethnicity (Rossetti et al., 2011; Tan et al., 2014; Wong et al., 2015), race (Goldstein et al., 2014; Tan et al., 2014; O'Driscoll and Shaikh, 2017), and (rural)habitat (Goldstein et al., 2014; Hilgeman et al., 2019) are known factors, others debate that these factors are better represented by 'literacy in late life' (Sisco et al., 2015). More important for our setting are the negative influence of substance abuse (Rojo-Mota et al., 2013; Pugh et al., 2018) or psychiatric diseases (Musso et al., 2014; Gierus and Mosiolek, 2015; Blair et al., 2016; Srisurapanont et al., 2016; Wu et al., 2017; Korsnes, 2020).

The above enumeration shows that there are many reasons for heterogeneity affecting the MoCA score. It shows that a single cutoff rarely fits a pluriform clinical practice where many covariates influence the individual MoCA score. A single cutoff is associated with substantially high rates of misclassification. Stratification was suggested for age and education as a solution (Oren et al., 2014; Wong et al., 2015; Borland et al., 2017). However, stratification of patients is impracticable if one needs to take all the possible confounders into account.

Psychiatric comorbidities, such as depression or mania, only substantially influence the MoCA score in some individuals (Gierus and Mosiolek, 2015; Blair et al., 2016; Dautzenberg et al., 2021; Korsnes, 2020), making beforehand stratification of these patients infeasible. This underscores that the MoCA should not be used as a stand-alone or overrule but should help clinical judgment as an add-on by knowing its strength (NVP, sensitivity) and weakness (PPV, specificity).

A strength of our study is that possible psychiatric causes of CI were not excluded, as (cloaked) psychiatry is the clinical reality in most settings, especially in old-age psychiatry. Another strength is that we used a clinical cohort setup by avoiding the extremes of the spectrum, such as community-based healthy controls, and severely demented including those with BPSD, following the STARDdem recommendations. Therefore, the cohort consisted of patients a clinician would consider screening for CI. Including severe dementia would give better results due to a higher dementia prevalence and lower MoCA scores, but this is not the clinical reality.

Our setting, on the other hand, is also a limitation, as referrals with BPSD and MCI caused by psychiatric etiology will be higher than those in nonpsychiatric settings and will influences the prevalence of MD. However, our previous study showed that the mean MoCA score did not differ between neurodegenerative and psychiatric etiologies of MCI (Dautzenberg et al., 2021).

A limitation of our study is the uncertainty of the number of FNs after initial assessment. The consecutive cohort design resulted in 'unsuspected patients after initial assessment' $(n=403)$ not receiving an NPA due to practicality and resource constraints. We minimized this flaw by following these patients for at least 2 years. Three out of 403 unsuspected patients progressed to CI, most likely new cases. This corresponds with the incidence of 6.6 males or 7.4 females in the $74-79$ age group (Volksgezondheidenzorg.info, 2019). Nonetheless, we labeled them as if they were FN at the initial assessment. As stated before, clinicians tend to refer when in doubt to minimize their FNs, which adds to their low FNs in this study. This favors the add-on strategy, but it also mimics the clinical reality.

The acceptance of the amount of FN next to the availability of an NPA will influence where one puts 
the cutoff for referring to an NPA. Simply changing a single cutoff will not improve the number of classification errors (Landsheer, 2020). With a second cutoff for monitoring, one can consider the pros and cons of lowering sensitivity against the gain of specificity but avoid absolute or binary decision errors. We also considered the use of a third cutoff, meaning below a certain score no referral is necessary as dementia is surely identified, i.e. diagnosed, but this is not feasible. Even with high dementia prevalence (e.g. including the severe or known demented, although not the clinical reality), the PPV of this extra cutoff would increase but never to the needed PPV of $100 \%$.

For settings similar to ours, we recommend the use of the double threshold as described of $<21$ and $\geq 26$, as these cutoffs give the best results, including the Youden index (Dautzenberg et al., 2020, 2021). This corresponds with the most error-prone scores of the MoCA (Landsheer, 2020) and is consistent with another study in old-age psychiatry, in which $87 \%$ of patients with dementia scored $<20$ and $100 \%$ scored $<23$ on the MoCA (Korsnes, 2020). In addition, almost all MCI patients with low MoCA scores $(<20)$ will develop MD, whereas only half of the MCI patients above this score will convert in the near future (Julayanont et al., 2014). Another study showed that $65 \%$ of their MCI patients with a score $<26$ did not convert to MD (Smith et al., 2007). This suggests that a double-threshold MoCA can separate low-risk MCI patients from very high-risk patients along with almost all patients with $\mathrm{MD}$ and benefit from a specialized diagnostic route.

Although our findings are not compatible with other settings, different settings may also benefit from a double-threshold MoCA. Whether it is to improve accuracy or because these settings have a more diverse population (and less uniformly distributed cognitive functioning). Even if one does not agree with our proposed policy because of a low prevalence of psychiatric diseases or easy access to specialized diagnostic routes in their setting. The three policies can easily be altered to fit once own setting, e.g. full memory clinic work up $<21 ; 21<$ active monitoring with an NPA $<26$; and $\geq 26$ watchful waiting with an MoCA.

As the MoCA can detect changes over time in MCI patients (Krishnan et al., 2017) and remains stable among cognitively normal patients (MalekAhmadi et al., 2018), active monitoring $(21<26)$ can be done by reassessment with a MoCA, which has three versions avoiding a learning curve (Costa et al., 2012; Nasreddine and Patel, 2016) and has a high retest reliability (Bruijnen et al., 2020). Together with an interview on IADL, giving an improved model fit (Durant et al., 2016) combined with an IQcode (De Jonghe, 1997), it can be administered in less than 30 minutes and could increase the overall diagnostic accuracy (Roalf et al., 2013). The average time of an NPA was $9 \mathrm{~h}$, including processing and feedback, at a cost of (in the Netherlands) $€ 110 / \mathrm{h}$. Therefore, the MoCA can not only reduce the stressful NPA waiting list but also avoid $€ 1000$ per FP and actively monitor those at risk less expensively.

To conclude, the optimal strategy for NPA referral is a two-stage selection process using the MoCA with a double threshold as an add-on after initial assessment. By selecting who is likely to have dementia and should be assessed further $(\mathrm{MoCA}<21)$, who should be discharged $(\geq 26)$, and who's course should be monitored actively as they are at risk $(21<26)$. This strategy not only gives the best results (accuracy, PPV, NPV) by referring most MD patients and reduces unnecessary FP referrals by $65 \%$. It also identifies most MCIs whose intermediate state justifies active monitoring. By introducing a second cutoff, the clinical value of the MoCA improved.

\section{Conflict of interest}

The authors have no conflict of interest.

\section{Description of authors' roles}

Géraud M. F. C. Dautzenberg: designed the study, collected the data, analyzing the data, and writing the paper.

Jeroen G. Lijmer: designed the study, assisted with writing the paper, supervized the data collection and statistical analyzing.

Aartjan T. F. Beekman: supervized the design and writing.

\section{Acknowledgments}

The authors thank Kasper Mens for his help to retrieve the digital data. The authors thank research assistants Andrea Hagg-Koelewijn, Ilse Geurts, Michelle Baars, Veerle van Meijl, Lara Verbeek, Sarah Rooijackers, Mariam Shanurkeyl, Wanisha Latchmansing, and Cagla Celik for their help collecting and digitalizing the test results and they also thank Jamie Nolan for his help in editing the manuscript.

\section{References}

Alzheimer's Disease International. (2018). From plan to impact Progress towards targets of the Global action plan on dementia. Available at: https://www.alz.co.uk/adi/pdf/fromplan-to-impact-2018.pdf. 
American Psychiatric Association. (2000). Diagnostic and Statistical Manual of Mental Disorders (revised 4th ed.). Washington, DC: American Psychiatric Association.

American Psychiatric Association. (2013). Diagnostic and Statistical Mental Disorders (DSM 5) (5th ed.). Arlington, VA: American Psychiatric Association.

Batelaan, N. M. et al. (2007). Thresholds for health and thresholds for illness: Panic disorder versus subthreshold panic disorder. Psychological Medicine, 37(2), 247-256. https://doi.org/10.1017/S0033291706009007.

Blair, M. et al. (2016). Depressive symptoms negatively impact montreal cognitive assessment performance: A memory clinic experience. Canadian fournal of Neurological Sciences, 43(4), pp. 513-517. https://doi.org/10.1017/cjn .2015 .399 .

Borland, E. et al. (2017). The Montreal cognitive assessment: Normative data from a large swedish population-based cohort. Fournal of Alzheimer's Disease, 59(3), pp. 893-901. https://doi.org/10.3233/ JAD-170203.

Borson, S. et al. (2013). Improving dementia care: The role of screening and detection of cognitive impairment. In Alzheimer's and Dementia (pp. 151-159) Elsevier Inc. https://doi.org/10.1016/j.jalz.2012.08.008.

Bossuyt, P. M. et al. (2015). STARD 2015: An updated list of essential items for reporting diagnostic accuracy studies. Clinical Chemistry, 61(12), 1446-1452. https://doi.org/10. 1373/clinchem.2015.246280.

Bruijnen, C. J. W. H. et al. (2020). Psychometric properties of the Montreal Cognitive Assessment (MoCA) in healthy participants aged 18-70. International fournal of Psychiatry in Clinical Practice, 24(3), 293-300. https://doi.org/10.1080/ 13651501.2020 .1746348$.

Burn, A. M. et al. (2018). Dementia case-finding in hospitals: A qualitative study exploring the views of healthcare professionals in English primary care and secondary care. BMF Open, 8(3), e020521. https://doi.org/ 10.1136/bmjopen-2017-020521.

Canevelli, M. et al. (2016). Spontaneous reversion of mild cognitive impairment to normal cognition: A systematic review of literature and meta-analysis. Fournal of the American Medical Directors Association, 17(10), 943-948. https://doi.org/10.1016/j.jamda.2016.06.020.

Carson, N., Leach, L. and Murphy, K. J. (2018) 'A reexamination of Montreal Cognitive Assessment (MoCA) cutoff scores', International fournal of Geriatric Psychiatry, 33(2), 379-388. https://doi.org/10.1002/gps.4756.

Costa, A. S. et al. (2012). Alternate-form reliability of the montreal cognitive assessment screening test in a clinical setting. Dementia and Geriatric Cognitive Disorders, 33(6), 379-384. https://doi.org/10.1159/000340006.

Dautzenberg, G., Lijmer, J. and Beekman, A. (2020). Diagnostic accuracy of the Montreal Cognitive Assessment (MoCA) for cognitive screening in old age psychiatry: Determining cutoff scores in clinical practice. Avoiding spectrum bias caused by healthy controls. International Fournal of Geriatric Psychiatry, 35(3), 261-269. https://doi .org/10.1002/gps.5227.

Dautzenberg, G., Lijmer, J. and Beekman, A. (2021). Clinical value of the Montreal Cognitive Assessment (MoCA) in patients suspected of cognitive impairment in old age psychiatry. Using the MoCA for triaging to a memory clinic. Cognitive Neuropsychiatry, 26(1), 1-17. https://doi.org/10.1080/13546805.2020.1850434.

Davis, D. H. J. et al. (2015). Montreal Cognitive Assessment for the diagnosis of Alzheimer's disease and other dementias. Cochrane Database of Systematic Reviews. https://doi.org/10.1002/14651858.CD010775.pub2.

Durant, J. et al. (2016). Relationship between the Activities of Daily Living Questionnaire and the Montreal Cognitive Assessment. Alzheimer's and Dementia: Diagnosis, Assessment and Disease Monitoring, 4, 43-46. https://doi.org/ 10.1016/j.dadm.2016.06.001.

Folstein, M. F., Folstein, S. E. and McHugh, P. R. (1975) "Mini-mental state". A practical method for grading the cognitive state of patients for the clinician. Fournal of Psychiatric Research, 12(3), 189-198. https://doi.org/10. 1016/0022-3956(75)90026-6.

Gauthier, S. et al. (2006). Mild cognitive impairment. Lancet, 1262-1270. https://doi.org/10.1016/S01406736(06)68542-5.

Gierus, J. and Mosiolek, A. (2015). The Montreal Cognitive Assessment as a preliminary assessment tool in general psychiatry Validity. Validity of MoCA in Psychiatric patients. General Hospital Psychiatry, 37, 476-480. https://doi .org/10.1016/j.genhosppsych.2015.05.011.

Glas, A. S. et al. (2003). The diagnostic odds ratio: a single indicator of test performance. Fournal of Clinical Epidemiology, 56(11), 1129-35. Available at: http://www. ncbi.nlm.nih.gov/pubmed/14615004; last accessed 15 April 2019

Goldstein, F. C. et al. (2014). Validity of the montreal cognitive assessment as a screen for mild cognitive impairment and dementia in African Americans. Fournal of Geriatric Psychiatry and Neurology, 27(3), 199-203. https:// doi.org/10.1177/0891988714524630.

Helmchen, H. and Linden, M. (2000). Subthreshold disorders in psychiatry: Clinical reality, methodological artifact, and the double-threshold problem. Comprehensive Psychiatry, 41(2), 1-7. https://doi.org/10.1016/S0010$440 \mathrm{X}(00) 80001-2$.

Hilgeman, M. et al. (2019). Use of the Montreal Cognitive Assessment (MoCA) in a rural outreach program for military veterans. Fournal of Rural Social Sciences, 34(2), Article 2. Available at: https://egrove.olemiss.edu/jrss/vol34/ iss $2 / 2$.

Ihara, M. et al. (2013). Association of physical activity with the visuospatial/executive functions of the montreal cognitive assessment in patients with vascular cognitive impairment. Fournal of Stroke and Cerebrovascular Diseases, 22(7), e146-e151. https://doi.org/10.1016/j. jstrokecerebrovasdis.2012.10.007.

Innocenti, A. et al. (2017). Lifestyle, physical activity and cognitive functions: The impact on the scores of Montreal Cognitive Assessment (MoCA). Archives Italiennes de Biologie, 155(1-2), 25-32. https://doi.org/10.12871/ 000398292017123.

Janssen, J. et al. (2019). Depressive symptoms and quality of life after screening for cognitive impairment in patients with type 2 diabetes: Observations from the Cog-ID cohort study. BMF Open, 9(1), e024696. https://doi.org/10.1136/ bmjopen-2018-024696.

De Jonghe, J. F. M. (1997). Differentiating between demented and psychiatric patients with the Dutch version of 
the IQCODE. International fournal of Geriatric Psychiatry, 12(4), 462-465. https://doi.org/10.1002/(SICI)10991166(199704)12:4<462:AID-GPS510>3.0.CO;2-Q.

Julayanont, P. et al. (2014). Montreal Cognitive Assessment Memory Index Score (MoCA-MIS) as a predictor of conversion from mild cognitive impairment to Alzheimer's disease. Fournal of the American Geriatrics Society, 62(4), 679-684. https://doi.org/10.1111/jgs.12742.

Korsnes, M. S. (2020). Performance on the mini-mental state exam and the Montreal cognitive assessment in a sample of old age psychiatric patients. SAGE Open Medicine, 8, 205031212095789. https://doi.org/10.1177/ 2050312120957895.

Krishnan, K. et al. (2017). Changes in Montreal cognitive assessment scores over time. Assessment, 24(6), 772-777. https://doi.org/10.1177/1073191116654217.

Landsheer, J. A. (2020). Impact of the prevalence of cognitive impairment on the accuracy of the Montreal cognitive assessment: The advantage of using two MoCA thresholds to identify error-prone test scores. Alzheimer Disease and Associated Disorders, 34(3), 248-253. https://doi. org/10.1097/WAD.0000000000000365.

Malek-Ahmadi, M. et al. (2018). Trajectory and variability characterization of the Montreal cognitive assessment in older adults. Aging Clinical and Experimental Research, 30(8), 993-998. https://doi.org/10.1007/s40520-017-0865-x.

mocatest.org. (no date). Available at: https://www.mocatest. org/; accessed 31 December 2019

Musso, M. W. et al. (2014). Investigation of the Montreal Cognitive Assessment (MoCA) as a cognitive screener in severe mental illness. Psychiatry Research, 220(1-2), 664-668. https://doi.org/10.1016/j.psychres.2014.07.078.

Nasreddine, Z. S. et al. (2005). The Montreal Cognitive Assessment, MoCA: A brief screening tool for mild cognitive impairment. Fournal of the American Geriatrics Society, 53(4), 695-699. https://doi.org/10.1111/j.15325415.2005.53221.x.

Nasreddine, Z. S. and Patel, B. B. (2016). Validation of Montreal Cognitive Assessment, MoCA, alternate french versions. Canadian fournal of Neurological Sciences, 43(5), 665-671. https://doi.org/10.1017/cjn.2016.273.

Nederlandse Vereniging voor Klinische Geriatrie. (2014). Richtlijn 'Diagnostiek en medicamenteuze behandeling van dementie'. Utrecht: CBO. Available at: https://www .nvvp.net/stream/richtlijn-diagnostiek-en-behandeling-vandementie-2014.

Noel-Storr, A. H. et al. (2014). Reporting standards for studies of diagnostic test accuracy in dementia: The STARDdem Initiative. Neurology, 83(4), 364-373. https:// doi.org/10.1212/WNL.0000000000000621.

O'Driscoll, C. and Shaikh, M. (2017). Cross-Cultural Applicability of the Montreal Cognitive Assessment (MoCA): A systematic review. fournal of Alzheimer's Disease. Edited by G. Chopard, 58(3), 789-801. https://doi .org/10.3233/JAD-161042.

Oren, N. et al. (2014). The montreal cognitive assessment in cognitively-intact elderly: A case for age-adjusted cutoffs. Fournal of Alzheimer's Disease, 43(1), 19-22. https://doi.org/ 10.3233/JAD-140774.

Pendlebury, S. T. et al. (2015). Routine cognitive screening in older patients admitted to acute medicine: Abbreviated mental test score (AMTS) and subjective memory complaint versus Montreal Cognitive Assessment and IQCODE. Age and Ageing, 44(6), 1000-1005. https://doi .org/10.1093/ageing/afv134.

Pinto, T. C. C. et al. (2018). Influence of age and education on the performance of elderly in the Brazilian version of the Montreal cognitive assessment battery. Dementia and Geriatric Cognitive Disorders, 45(5-6). https://doi.org/10. $1159 / 000489774$.

Pinto, T. C. C. et al. (2019). Is the Montreal Cognitive Assessment (MoCA) screening superior to the Mini-Mental State Examination (MMSE) in the detection of mild cognitive impairment (MCI) and Alzheimer's Disease (AD) in the elderly?. International Psychogeriatrics, 491-504. https://doi.org/10.1017/S1041610218001370.

Pugh, E. A. et al. (2018). Effects of normative adjustments to the montreal cognitive assessment. American fournal of Geriatric Psychiatry, 26(12), 1258-1267. https://doi.org/10. 1016/j.jagp.2018.09.009.

Reisberg, B. et al. (1982). The global deterioration scale for assessment of primary degenerative dementia. American fournal of Psychiatry, 139(9), 1136-1139. https://doi.org/ 10.1176/ajp.139.9.1136.

Roalf, D. R. et al. (2013). Comparative accuracies of two common screening instruments for classification of Alzheimer's disease, mild cognitive impairment, and healthy aging. Alzheimer's and Dementia, 9(5), 529-537. https://doi.org/10.1016/j.jalz.2012.10.001.

Rojo-Mota, G. et al. (2013). Neurocognitive screening in substance addicts: The Montreal Cognitive Assessment. Revista de Neurologia, 56(3), 129-136. https://doi.org/10. 33588/rn.5603.2012628.

Rossetti, H. C. et al. (2011). Normative data for the Montreal Cognitive Assessment (MoCA) in a populationbased sample. Neurology, 77(13), 1272-1275. https://oi. org/10.1212/WNL.0b013e318230208a.

Ryu, S. Y. et al. (2020). Self- and informant-reported cognitive functioning and awareness in subjective cognitive decline, mild cognitive impairment, and very mild Alzheimer disease', International fournal of Geriatric Psychiatry, 35(1), 91-98. https://doi.org/10.1002/gps.5224.

Schouws, S. N. T. M. et al. (2012). Self-reported cognitive complaints in elderly bipolar patients. American fournal of Geriatric Psychiatry, 20(8), 700-706. https://doi.org/10 .1097/JGP.0b013e31822ccd27.

Sisco, S. et al. (2015). The role of early-life educational quality and literacy in explaining racial disparities in cognition in late life. Journals of Gerontology - Series B Psychological Sciences and Social Sciences. Gerontological Society of America, 70(4), 557-567. https://doi.org/10. 1093/geronb/gbt133.

Smith, T., Gildeh, N. and Holmes, C. (2007). The Montreal cognitive assessment: Validity and utility in a memory clinic setting. Canadian fournal of Psychiatry. Canadian Psychiatric Association, 52(5), 329-332. https:// doi.org/10.1177/070674370705200508.

Srisurapanont, M. et al. (2016). Internal consistency and concurrent validity of the Montreal Cognitive Assessment in individuals with major depressive disorder. European Neuropsychopharmacology, 26, S384-S385. https://doi.org/ 10.1016/s0924-977x(16)31336-0. 
Swartz, R. H. et al. (2016). Validating a pragmatic approach to cognitive screening in stroke prevention clinics using the Montreal cognitive assessment. Stroke, 47(3), 807-813. https://doi.org/10.1161/STROKEAHA.115.011036.

Tan, J. P. et al. (2014). Optimal cutoff scores for dementia and mild cognitive impairment of the Montreal cognitive assessment among elderly and oldest-old Chinese population. Fournal of Alzheimer's Disease, 43(4), 1403-1412. https://doi.org/10.3233/JAD-141278.

Thomann, A. E. et al. (2020). Enhanced diagnostic accuracy for neurocognitive disorders: A revised cut-off approach for the Montreal Cognitive Assessment. Alzheimer's Research and Therapy, 12(1). https://doi.org/10. 1186/s13195-020-00603-8.

Volksgezondheidenzorg.info. (2019). Dementia numbers and context. Available at: https://www.volksgezondheidenzorg .info/onderwerp/dementie/cijfers-context/huidigesituatie\#node-aantal-nieuwe-gevallen-naar-leeftijd; accessed 20 January 2020
Winblad, B. et al. (2004). Mild cognitive impairment Beyond controversies, towards a consensus: Report of the International Working Group on Mild Cognitive Impairment. Fournal of Internal Medicine, 240-246. https://doi.org/10.1111/j.1365-2796.2004 $.01380 . \mathrm{x}$.

Wong, A. et al. (2015). Montreal cognitive assessment: One cutoff never fits all. Stroke, 46(12), 3547-3550. https://doi.org/10.1161/STROKEAHA.115.011226.

Wu, C., Dagg, P. and Molgat, C. (2017). Measuring stability of cognitive impairment in inpatients with schizophrenia with alternate forms of the Montreal Cognitive Assessment during acute hospitalization. Psychiatry Research, 258, 299-304. https://doi.org/10. 1016/j.psychres.2017.08.065.

Yesavage, J. A. and Sheikh, J. I. (1986). Geriatric Depression Scale (GDS): Recent evidence and development of a shorter version. Clinical Gerontologist, 5(1-2), 165-173. https://doi.org/10.1300/J018v05n01_09. 